\title{
RECEDING-HORIZON CONTROL FOR LINEAR TIME-DELAY SYSTEMS
}

\author{
Young Sam Lee* \\ * School of Electrical Engineering, Inha University, Inchon, \\ Korea
}

\begin{abstract}
Improved results on receding horizon control (RHC), or model predictive control (MPC), for linear state-delay systems is proposed. The proposed RHC has guaranteed closed-loop stability for wider class of state-delay systems than exiting RHC for state-delay systems. The proposed RHC is obtained by minimizing a new cost function that includes three terminal weighting terms, which are closely related to the closed-loop stability. Firstly, the solution of the proposed RHC is derived using the variational approach. Secondly, a delay-dependent matrix inequality condition to find stability-guaranteeing terminal weighting matrices is proposed. Simulation study shows that the proposed RHC has guaranteed closedloop stability even for a state-delay system that cannot be handled by the existing RHC. Copyright (C)2005 IFAC
\end{abstract}

Keywords: Receding horizon control (RHC), Delay-dependent matrix inequality, State-delay systems, Terminal weighting matrices

\section{INTRODUCTION}

In industrial processes, time-delays often occur in the transmission of material or information between different parts of a system. Chemical processing systems, transportation systems, communication systems, and power systems are typical examples of time-delay systems. Because the presence of a time-delay often causes serious deterioration of the stability and performance of the system, considerable research has been devoted to the control of time-delay systems.

For delay-free systems, the receding-horizon control (RHC), or model predictive control (MPC), has received considerable attention (Kwon and Pearson, 1977; Richalet et al., 1978; Kwon and Kim, 2000) because of its many advantages, including ease of computation, good tracking performance and I/O constraint handling capability, compared with the popular steady-state infinite horizon linear quadratic (LQ) control. While the steady-state LQ control for linear systems is obtained from the algebraic Riccati equation, the RHC for linear systems is obtained from the differential or difference Riccati equation on a finite interval, which is easier to solve. Therefore, RHC has been widely used, particularly in the chemical process industries (Richalet et al., 1978).

To the best of the authors' knowledge, there are two previous results on the RHC for state-delayed systems. A simple control method based on the receding horizon concept appears in (Kwon et $a l .$, 2003). However, it does not have a state weighting in the cost function. Furthermore, it does not guarantee closed-loop stability by design, and therefore stability can be checked only after the controller has been designed. The method proposed in (Kwon et al., 2004) has both state and input weighting terms in the cost function. Furthermore, it has guaranteed closed-loop stability by design. However, the method of (Kwon et al., 2004) has a drawback that only a limited 
class of state-delay systems has guaranteed closedloop stability. This implies that we can't apply the RHC of (Kwon et al., 2004) to a certain class of state-delay systems.

In RHC, terminal weighting matrices in the cost function are crucial for stability as already shown in (Kwon et al., 2004). The the cost function taken in (Kwon et al., 2004) includes two terminal weighting matrices and the LMI condition provided there to obtain stability-guaranteeing terminal weighting matrices is delay-independent. Because the condition is delay-independent, some class of state-delay systems does not satisfy it. Therefore, stability-guaranteeing terminal weighting matrices are not available. The cost function taken in this paper has three terminal weighting matrices in order to enlarge the class of statedelay systems that can be handled through the RHC. The proposed matrix condition used to find stability-guaranteeing terminal weighting matrices includes the delay information. As a consequence, the proposed RHC can handle state-delay systems that can't be through the conventional RHC of (Kwon et al., 2004).

\section{RECEDING HORIZON CONTROL FOR STATE-DELAY SYSTEMS}

Consider a time delay system

$$
\dot{x}(t)=A x(t)+A_{1} x(t-h)+B u(t)
$$

with the initial condition

$$
x(\tau)=\phi(\tau), \tau \in[-h, 0],
$$

where $x(t) \in \mathbf{R}^{n}$ is the state, $u(t) \in \mathbf{R}^{m}$ is the input, and $A, A_{1} \in \mathbf{R}^{n \times n}$, and $B \in \mathbf{R}^{n \times m}$ are system matrices. $h>0$ is the delay, and $\phi(t)$ is a continuous function. In order to obtain a receding horizon control, we first consider a finite horizon cost function represented by

$$
\begin{aligned}
& J\left(x_{t_{0}}, u, t_{0}, t_{f}\right) \\
& =\int_{t_{0}}^{t_{f}}\left[x^{T}(\tau) Q x(\tau)+u^{T}(\tau) R u(\tau)\right] d \tau \\
& \quad+x^{T}\left(t_{f}\right) F_{1} x\left(t_{f}\right)+\int_{t_{f}-h}^{t_{f}} x^{T}(\alpha) F_{2} x(\alpha) d \alpha \\
& \quad+\int_{-h}^{0} \int_{t_{f}+\beta}^{t_{f}} \dot{x}^{T}(\alpha) F_{3} \dot{x}(\alpha) d \alpha d \beta,
\end{aligned}
$$

where $x_{t_{0}}=x\left(t_{0}+s\right), s \in[-h, 0]$, is a continuous function, $t_{0}$ is the initial time, $t_{f}$ is the terminal time, and $Q>0, R>0, F_{1}>0, F_{2}>0$, and $F_{3}>$ 0. $t_{f}-t_{0}>0$ is the horizon length. It is noted that the cost function (2) has three terminal weighting terms. The optimal control minimizing the cost function (2) and the corresponding optimal cost will be denoted by $u^{*}(\tau), t_{0} \leq \tau \leq t_{f}$, and $J^{*}\left(x_{t_{0}}, t_{0}, t_{f}\right)$, respectively. It is apparent that $J^{*}\left(x_{t_{0}}, t_{0}, t_{f}\right)=J\left(x_{t_{0}}, u^{*}, t_{0}, t_{f}\right)$. The RHC is then obtained by minimizing the cost function (2) with the initial time $t_{0}$ and the terminal time $t_{f}$ replaced by the current time $t$ and $t+T_{p}$, respectively, where $T_{p}$ is a (prediction) horizon length that is a positive constant. As will be shown later, stability of the proposed $\mathrm{RHC}$ depends on the choice of three terminal weighting matrices $F_{1}$, $F_{2}$, and $F_{3}$.

In (Kwon et al., 2004), RHC minimizing the following cost function with $F_{3}=0$ has been proposed. However, only a limited class of statedelay systems satisfies the LMI condition on the stability-guaranteeing terminal weighting matrices provided in (Kwon et al., 2004) with $F_{3}=0$. This is because the condition is delayindependent. As will be seen in the next section, we can derive delay-dependent condition on stability-guaranteeing terminal weighting matrices by introducing the third weighting terms parameterized by $F_{3}$. This, in turn, enables the proposed RHC to handle wider class of statedelay systems than the existing RHC of (Kwon et al., 2004).

Firstly, the solution to the finite horizon optimal control problem will be derived. Secondly, RHC will be constructed from the derived solution. Throughout this paper, we assume $t_{f}-t_{0} \leq h$. In $\mathrm{RHC}, t_{f}-t_{0}$ reduces to the prediction horizon length $T_{p}$. Because $T_{p}$ is a design parameter, it can chosen such that $T_{p} \leq h$.

Noting $t_{f}-t_{0} \leq h$ and

$$
\begin{aligned}
& \int_{-h}^{0} \int_{t_{f}+\beta}^{t_{f}} \dot{x}^{T}(\alpha) F_{3} \dot{x}(\alpha) d \alpha d \beta \\
& =\int_{t_{f}-h}^{t_{f}}\left(\alpha-t_{f}+h\right) \dot{x}^{T}(\alpha) F_{3} \dot{x}(\alpha) d \alpha
\end{aligned}
$$

the cost function (2) is rewritten as

$$
J\left(x_{t_{0}}, u, t_{0}, t_{f}\right)=\bar{J}\left(x_{t_{0}}, u, t_{0}, t_{f}\right)+\Omega
$$

where

$$
\begin{aligned}
\bar{J}\left(x_{t_{0}}, u, t_{0}, t_{f}\right) \\
=\int_{t_{0}}^{t_{f}}\left[x^{T}(\tau) \bar{Q}(\tau) x(\tau)+2 x^{T}(\tau) N(\tau) u(\tau)\right. \\
\quad+u^{T}(\tau) \bar{R}(\tau) u(\tau)+2 x^{T}(\tau) U(\tau) x(\tau-h) \\
\left.\quad+2 u^{T}(\tau) V(\tau) x(\tau-h)\right] d t \\
\quad+x^{T}\left(t_{f}\right) F_{1} x\left(t_{f}\right), \\
\Omega=\int_{t_{f}-h}^{t_{0}}\left[x^{T}(\tau) F_{2} x(\tau)+f(\tau) \dot{x}(\tau) F_{3} \dot{x}(\tau)\right] d \tau \\
\quad+\int_{t_{0}}^{t_{f}} f(\tau) x^{T}(\tau-h) A_{1}^{T} F_{3} A_{1} x(\tau-h) d \tau
\end{aligned}
$$


and

$$
\begin{aligned}
& f(\tau) \triangleq\left(\tau-t_{f}+h\right) \\
& \bar{Q}(\tau) \triangleq Q+F_{2}+f(\tau) A^{T} F_{3} A, \\
& \bar{R}(\tau) \triangleq R+f(\tau) B^{T} F_{3} B, \\
& N(\tau) \triangleq f(\tau) A^{T} F_{3} B, \\
& U(\tau) \triangleq f(\tau) A^{T} F_{3} A_{1}, \\
& V(\tau) \triangleq f(\tau) B^{T} F_{3} A_{1} .
\end{aligned}
$$

It is noted that the term $\Omega$ is constant because the two integral terms are known. Therefore, instead of minimizing the original cost function $J\left(x_{t_{0}}, u, t_{0}, t_{f}\right)$, we minimize a new cost function $\bar{J}\left(x_{t_{0}}, u, t_{0}, t_{f}\right)$. It is apparent that the optimal control minimizing the cost function $\bar{J}\left(x_{t_{0}}, u, t_{0}, t_{f}\right)$ also minimizes the original cost function $J\left(x_{t_{0}}, u, t_{0}, t_{f}\right)$.

Given $x_{t_{0}}, x(\tau-h)$ for $t_{0} \leq \tau \leq t_{f}$ is a known term if $\left(t_{f}-t_{0}\right) \leq h$. Therefore we can use the existing variational approach of optimal control theory (Kirk, 1970) to obtain the optimal control for the state-delay system (1). The necessary and sufficient conditions for optimality are given as follows:

$$
\begin{aligned}
\dot{x}(\tau)= & A x(\tau)+A_{1} x(\tau-h)+B u(\tau), \\
\dot{p}(\tau)= & -\bar{Q}(\tau) x(\tau)-N(\tau) u(\tau) \\
& -U(\tau) x(\tau-h)-A^{T} p(\tau), \\
0= & N^{T}(\tau) x(\tau)+\bar{R}(\tau) u(\tau) \\
& +V(\tau) x(\tau-h)+B^{T} p(\tau), \\
p\left(t_{f}\right)= & F_{1} x\left(t_{f}\right),
\end{aligned}
$$

for $t_{0} \leq \tau \leq t_{f}$, where $p(t)$ is the costate. Let us define

$$
\mathbf{H}(\tau) \triangleq\left[\begin{array}{ll}
H_{11}(\tau) & H_{12}(\tau) \\
H_{21}(\tau) & H_{22}(\tau)
\end{array}\right],
$$

where

$$
\begin{aligned}
& H_{11}(\tau)=A-B \bar{R}^{-1}(\tau) N^{T}(\tau) \\
& H_{12}(\tau)=-B \bar{R}^{-1}(\tau) B^{T} \\
& H_{21}(\tau)=-\bar{Q}(\tau)+N(\tau) \bar{R}^{-1}(\tau) N^{T}(\tau) \\
& H_{22}(\tau)=-A^{T}+N(\tau) \bar{R}^{-1}(\tau) B^{T} .
\end{aligned}
$$

Let $\Phi(\cdot, \cdot)$ be the state transition matrix of $H(\tau)$. $\Phi$ is partitioned into

$$
\Phi(\cdot, \cdot)=\left[\begin{array}{ll}
\Phi_{11}(\cdot, \cdot) & \Phi_{12}(\cdot, \cdot) \\
\Phi_{21}(\cdot, \cdot) & \Phi_{22}(\cdot, \cdot)
\end{array}\right] .
$$

From the optimal control theory (Kirk, 1970), we obtain

$$
p(\tau)=W_{1}(\tau) x(\tau)
$$

$$
+\int_{-h}^{t_{f}-\tau-h} W_{2}(\tau, s) x(\tau+s) d s,
$$

and the optimal control $u^{*}(\tau)$ for $t_{0} \leq \tau \leq t_{f}$

$$
\begin{aligned}
u^{*}(\tau)= & -\bar{R}^{-1}(\tau) B^{T}\left(\left[W_{1}(\tau)+f(\tau) F_{3} A\right] x(\tau)\right. \\
& +\int_{-h}^{t_{f}-\tau-h} W_{2}(\tau, s) x(\tau+s) d s \\
& \left.+f(\tau) F_{3} A_{1} x(\tau-h)\right)
\end{aligned}
$$

where

$$
\begin{aligned}
& W_{1}(\tau)=\left[\Phi_{22}\left(t_{f}, \tau\right)-F_{1} \Phi_{12}\left(t_{f}, \tau\right)\right]^{-1} \\
& \times\left[F_{1} \Phi_{11}\left(t_{f}, \tau\right)-\Phi_{21}\left(t_{f}, \tau\right)\right] \\
& W_{2}(\tau, s)=\left[\Phi_{22}\left(t_{f}, \tau\right)-F_{1} \Phi_{12}\left(t_{f}, \tau\right)\right]^{-1} \\
& \times\left\{\left[F_{1} \Phi_{11}\left(t_{f}, \tau+s+h\right)-\Phi_{21}\left(t_{f}, \tau+s+h\right)\right]\right. \\
& \times \bar{A}_{1}(\tau+s+h) \\
& +\left[F_{1} \Phi_{12}\left(t_{f}, \tau+s+h\right)-\Phi_{22}\left(t_{f}, \tau+s+h\right)\right] \\
& \left.\bar{A}_{2}(\tau+s+h)\right\} .
\end{aligned}
$$

In RHC, the initial time $t_{0}$ and terminal time $t_{f}$ correspond to $t$ and $t+T_{p}$, respectively. RHC is obtained when $\tau=t$. For fixed horizon length $T_{p}$, we can show that $W_{1}(t)$ and $W_{2}(t, s)$ is decided independently of $t$. Therefore, we denote $W_{1}(t)$ and $W_{2}(t, s)$ by $W_{1}$ and $W_{2}(s)$, respectively. Finally we obtain $\mathrm{RHC}$ as follows:

$$
\begin{aligned}
u^{*}(t)= & -\left[R+\left(h-T_{p}\right) B^{T} F_{3} B\right]^{-1} B^{T} \\
& \times\left\{\left[W_{1}+\left(h-T_{p}\right) F_{3} A\right] x(t)\right. \\
& +\int_{-h}^{T_{p}-h} W_{2}(s) x(t+s) d s \\
& \left.+\left(h-T_{p}\right) F_{3} A_{1} x(t-h)\right\} .
\end{aligned}
$$

In a special case of $T_{p}=h, \mathrm{RHC}$ is simplified to

$$
\begin{aligned}
u^{*}(t)= & -R^{-1} B^{T}\left[W_{1} x(t)\right. \\
& \left.+\int_{-h}^{0} W_{2}(s) x(t+s) d s\right] .
\end{aligned}
$$

In order to get $W_{1}(\tau)$ and $W_{2}(\tau, s)$ in (11) and (12), we have to determine the state transition matrix for $\mathbf{H}(\tau)$, which is often very tedious process. In the following theorem, we provide an alternative method to obtain $W_{1}(\tau)$ and $W_{2}(\tau, s)$ in terms of nonlinear matrix partial differential equations, which is solved backward in time.

Theorem 2.1. The matrix $W_{1}(\tau)$ and $W_{2}(\tau, s)$ satisfy the partial differential equations

$$
\dot{W}_{1}(\tau)-\left[W_{1}(\tau)+f(\tau) F_{3} A\right]^{T} B \bar{R}^{-1}(\tau) B^{T}
$$




$$
\begin{aligned}
& \times\left[W_{1}(\tau)+f(\tau) F_{3} A\right]+A^{T} W_{1}(\tau)+W_{1}(\tau) A \\
& +Q+F_{2}+f(\tau) A^{T} F_{3} A=0 \\
& \left(\frac{\partial}{\partial \tau}-\frac{\partial}{\partial s}\right) W_{2}(\tau, s)+A^{T} W_{2}(\tau, s) \\
& -\left[W_{1}(\tau)+f(\tau) F_{3} A\right]^{T} B \bar{R}^{-1}(\tau) B^{T} W_{2}(\tau, s)=0
\end{aligned}
$$

with boundary conditions

$$
\begin{aligned}
W_{2}(\tau,-h)= & W_{1}(\tau) A_{1}+f(\tau) A^{T} F_{3} A_{1} \\
& -f(\tau)\left[W_{1}(\tau)+f(\tau) F_{3} A\right]^{T} \\
& \times B \bar{R}(\tau)^{-1} B^{T} F_{3} A_{1} \\
W_{1}\left(t_{f}\right)= & F_{1}
\end{aligned}
$$

where $t_{0} \leq \tau \leq t_{f}$ and $-h \leq s \leq t_{f}-\tau-h$.

Proof. The proof is omitted due to the space limitation.

Remark 2.1. The partial differential equation in (16) looks difficult to solve at first glance. However, using the idea proposed in (Eller et al., 1969), we can change (16) into an equivalent ordinary differential equation that is easily solved.

\section{MONOTONIC CONDITION OF THE OPTIMAL COST}

In this section, we present a condition on the terminal weighting matrices $F_{1}, F_{2}$, and $F_{3}$, under which the optimal cost $J^{*}\left(x_{t_{0}}, t_{0}, \sigma\right)$ decreases while the terminal time $\sigma$ increases. We will call it a monotonic condition of the optimal cost on the terminal weighting matrices. As will be shown in the next section, the monotonic condition of the optimal cost is the sufficient condition for the closed-loop stability of systems controlled by the RHC. Before moving on, it is noted that $\star$ denotes the entries of a symmetric matrix, which are implied by symmetry.

Theorem 3.1. Assume that $F_{1}, F_{2}$, and $F_{3}$ in (2) satisfy the following matrix inequality for some $K$, $K_{1}, T_{1}, T_{2}, Y_{1}, Y_{2}, Y_{3}$ :

$$
\left[\begin{array}{cccc}
\Phi_{11} & \Phi_{12} & \Phi_{13} & h Y_{1} \\
\star & \Phi_{22} & \Phi_{23} & h Y_{2} \\
\star & \star & \Phi_{33} & h Y_{3} \\
\star & \star & \star & -h F_{3}
\end{array}\right]<0 .
$$

where

$$
\begin{aligned}
\Phi_{11} \triangleq & F_{2}+Q+K^{T} R K+Y_{1}+Y_{1}^{T}-T_{1}(A+B K) \\
& -(A+B K)^{T} T_{1}^{T} \\
\Phi_{12} \triangleq & F_{1}+Y_{2}^{T}+T_{1}-(A+B K)^{T} T_{2}^{T} \\
\Phi_{13} \triangleq & Y_{3}^{T}-Y_{1}-T_{1}\left(A_{1}+B K_{1}\right)+K^{T} R K_{1} \\
\Phi_{22} \triangleq & h F_{3}+T_{2}+T_{2}^{T} \\
\Phi_{23} \triangleq & -Y_{2}-T_{2}\left(A_{1}+B K_{1}\right)
\end{aligned}
$$

$\Phi_{33} \triangleq-F_{2}-Y_{3}-Y_{3}^{T}+K_{1}^{T} R K_{1}$.

The optimal cost $J^{*}\left(x_{t_{0}}, t_{0}, \sigma\right)$ then satisfies the following monotonic property:

$$
\frac{\partial J^{*}\left(x_{t_{0}}, t_{0}, \sigma\right)}{\partial \sigma}<0, \sigma>t_{0}
$$

Proof. The proof is omitted due to the space limitation.

Remark 3.1. It is noted that the case of $F_{3}=0$ corresponds to a cost function for the RHC proposed in (Kwon et al., 2004). As will be illustrated in a numerical example later in this paper, we can enlarge the class of state-delay systems that satisfy the monotonic condition of the optimal cost by adding the third terminal weighting terms parameterized by $F_{3}$.

We have to solve the matrix inequality (19) to obtain three terminal weighting matrices satisfying the monotonic condition of the optimal cost. However, the matrix inequality (19) in itself is hard to solve because it includes product terms of matrix variables. In the following theorem, we convert the matrix inequality (19) into an equivalent form which is easier to solve.

Theorem 3.2. Assume that there exist $L_{1}>0, L_{2}$, $L_{3}, N_{1}, N_{2}, N_{3}, V, V_{1}, U$ such that

$$
\left[\begin{array}{ccccccc}
\Gamma_{11} & \Gamma_{12} & \Gamma_{13} & h N_{1} & L_{1} Q^{\frac{1}{2}} & V^{T} R^{\frac{1}{2}} & h L_{2}^{T} \\
\star & \Gamma_{22} & \Gamma_{23} & h N_{2} & 0 & 0 & h L_{3}^{T} \\
\star & \star & \Gamma_{33} & h N_{3} & 0 & V_{1}^{T} R^{\frac{1}{2}} & 0 \\
\star & \star & \star & -h L_{1} U^{-1} L_{1} & 0 & 0 & 0 \\
\star & \star & \star & \star & -I & 0 & 0 \\
\star & \star & \star & \star & \star & -I & 0 \\
\star & \star & \star & \star & \star & \star & -h U
\end{array}\right]<0
$$

where

$$
\begin{aligned}
& \Gamma_{11}=L_{2}+L_{2}^{T}+N_{1}+N_{1}^{T}+W \\
& \Gamma_{12}=L_{3}+L_{2}^{T}-\left(A L_{1}+B V\right)^{T}+N_{2}^{T} \\
& \Gamma_{13}=-N_{1}+N_{3}^{T} \\
& \Gamma_{22}=L_{3}+L_{3}^{T} \\
& \Gamma_{23}=-\left(A_{1} L_{1}+B V_{1}\right)-N_{2} \\
& \Gamma_{33}=-W-N_{3}-N_{3}^{T} .
\end{aligned}
$$

Then the matrix inequality (19) of Theorem 3.1 is feasible and terminal weighting matrices satisfying the inequality (19) can be chosen such that $F_{1}=L_{1}^{-1}, F_{2}=L_{1}^{-1} W L_{1}^{-1}, F_{3}=U^{-1}$.

Proof. The feasibility of (19) requires $T_{2}+T_{2}^{T}<$ 0 , which in turn requires $T_{2}$ is nonsingular. Define

$$
\left[\begin{array}{cc}
F_{1} & 0 \\
T_{1}^{T} & T_{2}^{T}
\end{array}\right]^{-1}=L=\left[\begin{array}{cc}
L_{1} & 0 \\
L_{2} & L_{3}
\end{array}\right] .
$$


It is noted that $L$ is nonsingular if (19) is feasible. Pre- and post-multiply (19) by $\operatorname{diag}\left\{L^{T}, L_{1}, L_{1}\right\}$ and $\operatorname{diag}\left\{L, L_{1}, L_{1}\right\}$, respectively and introduce some change of variables such that

$L_{1} \triangleq F_{1}^{-1}, W \triangleq L_{1} F_{2} L_{1}, U \triangleq F_{3}^{-1}, V \triangleq K L_{1}$,

$V_{1} \triangleq K_{1} L_{1}, L^{T}\left[\begin{array}{l}Y_{1} \\ Y_{2}\end{array}\right] L_{1} \triangleq\left[\begin{array}{l}N_{1} \\ N_{2}\end{array}\right], L_{1} Y_{3} L_{1} \triangleq N_{3}$

The matrix inequality (19) is then equivalently changed to (21) through the Schur complement. This implies (19) is feasible if (21) is feasible. This completes the proof.

Remark 3.2. It is noted that (21) is not an LMI condition because of the term $-h L_{1} U^{-1} L_{1}$ and hence looks difficult to solve. However, using the method presented in (Lee et al., 2004), the matrix inequality (21) can be easily solved in an iterative manner through existing LMI solvers.

\section{STABILITY OF RHC}

This section investigates the stability of the RHC.

Theorem 4.1. Given $Q>0$ and $R>0$, if $\frac{\partial J^{*}\left(x_{t_{0}}, t_{0}, \sigma\right)}{\partial \sigma} \leq 0$ for $\sigma>t_{0}$, the system (1) controlled by the RHC is asymptotically stable.

Proof. See (Kwon et al., 2004).

Using the sufficient conditions for monotonic cost, the following theorem is obtained.

Theorem 4.2. Given $Q>0$ and $R>0$, assume that the matrix inequality (21) is feasible. Then the proposed RHC in (13) designed with terminal weighting matrices $F_{1}=L_{1}^{-1}, F_{2}=L_{1}^{-1} W L_{1}^{-1}$, $F_{3}=U^{-1}$ asymptotically stabilizes the system (1).

Proof. From Theorem 4.1, we see that $\frac{\partial J^{*}\left(x_{t_{0}}, t_{0}, \sigma\right)}{\partial \sigma} \leq 0$ for $\sigma>t_{0}$ is the sufficient condition for asymptotic stability of the system controlled by the RHC. Feasibility of (21) is equivalent to feasibility of (19). Therefore, if we choose terminal weighting matrices such that $F_{1}=L_{1}^{-1}$, $F_{2}=L_{1}^{-1} W L_{1}^{-1}, F_{3}=U^{-1}$, the optimal cost $J^{*}\left(x_{t_{0}}, t_{0}, \sigma\right)$ satisfies (20) and resultant RHC asymptotically stabilizes the system. This completes the proof.

\section{A NUMERICAL EXAMPLE}

In this section, we provide a numerical example to illustrate that the proposed RHC method can handle wider class of state-delay systems than existing RHC.

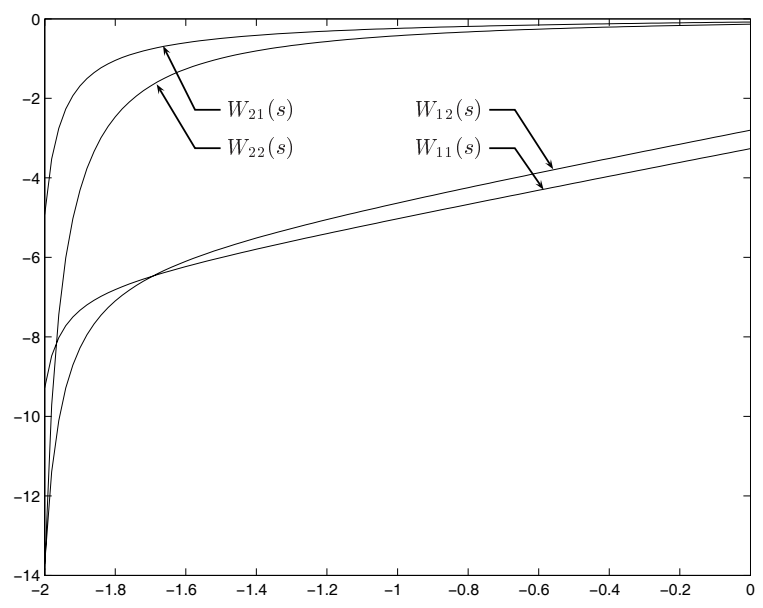

Fig. 1. Plot of $W_{2}(s)$

Consider a state-delay system whose system matrices and delay length are given by

$A=\left[\begin{array}{ll}0 & 0 \\ 0 & 1\end{array}\right], \quad A_{1}=\left[\begin{array}{rr}-1 & -1 \\ 0 & -0.9\end{array}\right], \quad B=\left[\begin{array}{l}0 \\ 1\end{array}\right], \quad h=2$.

The weighting matrices $Q$ and $R$ are chosen such that $Q=I$ and $R=I$. It can be checked through simulation that the system is open-loop unstable. It is noted that the RHC proposed in (Kwon et al., 2004) cannot be applied to this system because we can't find any stability-guaranteeing terminal weighting matrices using the LMI condition proposed there.

The first step for applying the proposed RHC is to find terminal weighting matrices $F_{1}, F_{2}$ and $F_{3}$ guaranteeing the closed-loop stability. Solving the matrix inequality (21) yields

$$
\begin{aligned}
& F_{1}=\left[\begin{array}{cc}
10.76 & 13.97 \\
\star & 26.94
\end{array}\right], F_{2}=\left[\begin{array}{cc}
8.08 & 13.41 \\
\star & 22.28
\end{array}\right], \\
& F_{3}=\left[\begin{array}{cc}
8.83 & 9.58 \\
\star & 20.41
\end{array}\right] .
\end{aligned}
$$

The second step is to obtain $W_{1}$ and $W_{2}(s)$ in (13) for given $T_{p}$ by solving (15) and (16). In case of $T_{p}=h$, RHC is represented by (14) with

$$
W_{1}=\left[\begin{array}{cc}
9.28 & 4.92 \\
\star & 9.93
\end{array}\right], W_{2}(s)=\left[\begin{array}{ll}
W_{11}(s) & W_{12}(s) \\
W_{21}(s) & W_{22}(s)
\end{array}\right] .
$$

Figure 1 shows plot of $W_{2}(s)$ for $-2 \leq s \leq 0$. In order to find how the horizon length affects the performance, we design three RHCs with the horizon lengths, $T_{p}=0.5,1.5$, and 2 and apply them to the system with initial state $\phi_{1}(\theta)=$ $1, \phi_{2}(\theta)=0,-h \leq \theta \leq 0$. To measure the performances of the designed RHCs, we use an integrated cost given by

$$
J_{I N T}=\int_{0}^{40}\left[x^{T}(t) Q x(t)+u^{T}(t) R u(t)\right] d t .
$$

Figure 2 compares the state trajectories of $x_{1}$ resulting from those three RHCs. We see that three RHCs are all stabilizing. It is noted that 


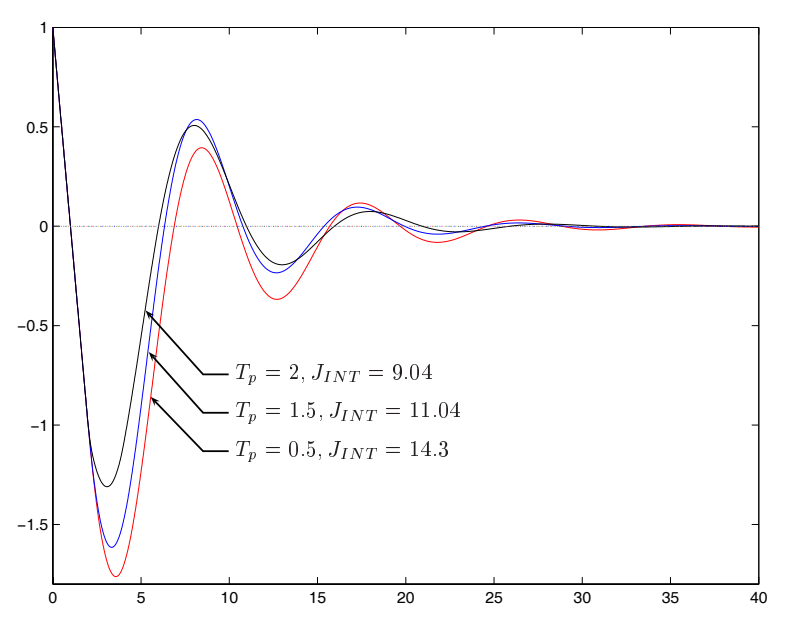

Fig. 2. State trajectories of $x_{1}$ due to RHCs with different horizon lengths

$J_{I N T}$ decreases as $T_{p}$ increases, which implies RHC with longer prediction horizon yields better performance. This in turn implies that we can change the controller performance by adjusting the horizon length.

From this example, we see that the proposed RHC has guaranteed closed-loop stability and can handle wider class of state-delay systems than the existing RHC in (Kwon et al., 2004).

\section{CONCLUSIONS}

A stabilizing RHC (or MPC) for linear statedelay systems was proposed in this paper. We proposed a new receding horizon cost function with three terminal weighting terms and derived the RHC minimizing that cost function. The solution of the RHC turned out to be parameterized by the solution to nonlinear matrix partial differential equations solved backward in time. A matrix inequality condition to obtain stabilityguaranteeing terminal weighting matrices for the $\mathrm{RHC}$ was proposed. The main contribution of the work is that the proposed RHC has guaranteed closed-loop stability for wider class of state-delay systems than the existing RHC for state-delay systems. It is regarded that the proposed method can be widely used for various processes with a statedelay, particularly for chemical processes that are usually slow in dynamics.

\section{REFERENCES}

Eller, D. H., J. K. Aggarwal and H. T. Banks (1969). Optimal control of linear time-delay systems. IEEE Trans. Automat. Contr. AC14(6), 678-687.

Kirk, D. E. (1970). Optimal Control Theory. Prentice-Hall.

\section{Prentice-Hall.}

Kwon, W. H. and A. Pearson (1977). A modified quadratic cost problem and feedback stabilization of a linear system. IEEE Trans. Automat. Contr. 32(5), 838-842.

Kwon, W. H. and K. B. Kim (2000). On stabilizing receding horizon controls for linear continuous time-invariant systems. IEEE Trans. Automat. Contr. 45(7), 1329-1334.

Kwon, W. H., Jin Won Kang, Young Sam Lee and Young Soo Moon (2003). Simple receding horizon control for State Delayed Systems with Its Stability. Journal of Process Control 13(6), 539-551.

Kwon, W. H., Y. S. Lee and S. H. Han (2004). General receding horizon control for linear time-delay systems. Automatica 40(9), 16031611.

Lee, Y. S., Y. S. Moon, W. H. Kwon and P. G. Park (2004). Delay-dependent robust $H_{\infty}$ control for uncertain systems with a statedelay. Automatica 40(1), 65-72.

Richalet, J., A. Rault, J. L. Testud and J. Papon (1978). Model predictive heuristic control: Applications to industrial processes. $A u$ tomatica 14, 413-428. 